\title{
Cas de coccidiose cæcale chez des poules pondeuses après diverses manipulations
}

\author{
A. Maho ${ }^{1}$ B. Ndobale ${ }^{2}$
}

\section{Mots-clés}

Volaille - Poule pondeuse - Coccidiose Eimeria tenella - Mortalité - Tchad.

\section{Résumé}

Suite à plusieurs manipulations effectuées le même jour sur une bande de poules pondeuses Lohmann brown dans une ferme située dans la banlieue de N'Djaména, une mortalité de 6,6 p. 100 a été enregistrée six jours après ces manipulations. Les autopsies et les analyses parasitologiques et bactériologiques ont permis d'identifier Eimeria tenella comme responsable et de conclure que les effets associés de ces manipulations ont baissé la résistance des oiseaux et occasionné l'apparition de cette pathologie.

\section{INTRODUCTION}

Les coccidies sont des protozoaires difficiles à éviter en élevage industriel, sévissant principalement sur de jeunes sujets et souvent lors d'altération de l'environnement. Toutefois, elles peuvent affecter des sujets adultes lorsque les conditions de leur développement dans l'organisme sont favorisées $(1,2,4,7)$. Ce sont probablement ces conditions qui ont permis aux coccidies d'affecter les poules pondeuses, objets de cette communication.

\section{MATERIEL ET METHODES}

Neuf cadavres et trois malades appartenant à une bande de 1252 poules pondeuses de race Lohmann brown âgées de neuf mois et provenant d'une ferme située en banlieue de N'Djaména ont été apportées au Laboratoire de recherches vétérinaires et zootechniques de Farcha. Cette mortalité fait suite aux débecquage, trempage et transfert des oiseaux dans un nouveau bâtiment, effectués le 20 septembre 1996, sans traitement préalable aux anti-stress. La mortalité a commencé trois jours après ces manipulations et a entrâné, en six jours, 83 morts (6,6 p. 100).

Au laboratoire, les oiseaux ont été autopsiés selon les procédures classiques : examens externes, puis internes. Les organes présentant des lésions ont été analysés parasitologiquement et bactériologiquement. En parasitologie, les matières fécales et la muqueuse

1. Laboratoire de Recherches Vétérinaires et Zootechniques de Farcha, BP 433, N'Djaména, Tchad

2. Ecole Nationale des Agents Techniques de l'Elevage, BP 750, N'Djaména, Tchad cæcale des douze oiseaux autopsiés ont été observées au microscope optique entre lame et lamelle et après réalisation de frottis frais, pour rechercher les parasites.

En bactériologie, les organes suspects ont été observés au microscope optique après la réalisation de frottis colorés au gram. Les organes présentant une bactérioscopie positive ont été cultivés sur les milieux d'isolement d'entérobactéries :

- milieu EMB (milieu de Levine);

- milieu MacConkey ;

- bouillon pasteurelle (pour la recherche de Pasteurella);

- bouillon sélénite (milieu enrichi pour la recherche de Salmonella). Après $24 \mathrm{~h}$ d'incubation, les cultures ont été isolées dans un milieu plus spécifique (milieu Shigella-Salmonella).

Après isolement, les cultures suspectes ont été identifiées à l'aide de la galerie Api20E.

RESU LTATS

Le résultat des examens macroscopiques, microscopiques et bactériologiques effectués sur les 3 malades et les 9 cadavres après autopsie sont présentés dans le tableau.

\section{Examens macroscopiques}

Huit poules présentaient un cloaque souillé de matières fécales striées de sang et quatre autres ne présentaient aucun signe de diarrhée. Sept oiseaux étaient maigres et les autres en état d'embonpoint satisfaisant. 
Tableau

Résultat des examens macroscopiques, microscopiques et bactériologiques effectués sur les 3 malades et les 9 cadavres après autopsie

\section{Lésions observées}

Typhlite

Hépatomégalie

Splénomégalie

Gonflement de l'intestin

Diarrhée

Maigreur

Présence des coccidies dans les matières fécales

Présence des coccidies dans la muqueuse cæcale

Présence d'Escherichia coli

Présence de Proteus mirabilis

\section{Nombre de cadavres positifs}

9

$4-2$

$3-1$

5

$7-1$

$6-1$

$8-2$

9

$3-0$

$3-1$

L'examen interne a mis en évidence une typhlite sur l'ensemble des oiseaux autopsiés. D'autres poules ont montré par ailleurs une splénomégalie (4 poules), une hépatomégalie (6 poules) et un gonflement de l'intestin (6 poules).

\section{Examens microscopiques}

\section{Parasitologie}

Les examens des matières fécales et de la muqueuse cæcale ont révélé la présence d'oocystes d'Eimeria tenella sur tous les oiseaux autopsiés. La présence très élevée de ce parasite était surtout remarquée sur la muqueuse des oiseaux sans diarrhée et en bon état d'embonpoint.

\section{Bactériologie}

Les cultures des organes suspects (foie, rate) et des matières fécales ont permis d'isoler Escherichia coli et Proteus mirabilis sur respectivement 3 et 4 cadavres.

\section{DISCUSSION}

La vaccination contre la coccidiose aviaire n'en est qu'à ses débuts et l'immunité naturelle en élevage industriel est souvent insuffisante ; toute agression par un agent pathogène ou par un stress d'élevage baisse la résistance des animaux (1). La mortalité constatée trois jours après les manipulations des oiseaux, alors que la période prépatente de ces protozooses varie de 4-7 jours, montre que les oiseaux hébergeaient déjà les coccidies mais de façon latente. En effet, il est rapporté qu'une forte immunité antiparasitaire entraîne une baisse, voire l'inhibition, d'excrétion d'oocystes d'Eimeria tenella tandis qu'une faible immunité augmente l'excrétion d'oocystes et baisse l'ingestion alimentaire, l'efficacité de la conversion alimentaire et le taux leucocytaire plasmatique (3, 6). Par ailleurs, Ugochukwu (6) décrit en 1982 une épidémie de coccidiose cæcale avec une sévère mortalité lors d'une vaccination intramusculaire contre la maladie de Newcastle. Oluigbo et Enurah (5) rapporte en 1989 une mortalité de 75 p. 100 lors d'une infection simultanée «maladie de Gumboro et coccidiose cæcale ». Le stress est probablement un facteur favorisant cette expression de la coccidiose. Il est donc dangereux de soumettre les oiseaux à plusieurs stress simultanés ou successifs, leurs effets pouvant s'additionner et déprimer l'état immunitaire des animaux. En outre, il serait prudent de faire précéder toute manipulation par l'administration d'un anti-stress.

McKee et Harrison (4) ont montré que l'administration d'acide ascorbique comme anti-stress avant le trempage des oiseaux, augmente l'ingestion, l'efficacité de la conversion alimentaire et limite la réduction du taux leucocytaire plasmatique.

\section{BIBLIO GRAPHIE}

1. BIESTER H.E., SCHWARTE L.H., 1965. Diseases of poultry. Ames, Iowa, USA, The lowa State U niversity Press, $1382 \mathrm{p}$.

2. BRUGERE J.P., SILMIN A., 1992. Manuel de pathologie aviaire. Maisons-Alfort, France, Ecole nationale vétérinaire d'Alfort, $381 \mathrm{p}$

3. TORTINEAU O., TRONCY P.N., 1985. Coccidiose, maladie animale majeure - II. Les coccidioses du poulet. Revue Elev. Méd. vét. Pays trop., N ouvelle Calédonie (6) : 9-17.

4. MCKEE J.S., HARRISO N P.C., 1995. Effect of supplemental ascorbic acid on the performance of broiler chickens exposed to multiple concurrent stress. Poult. Sci., 74 (11): 1972-1985.

5. OLUIGBO F., ENURAH L.U., 1989. Infection simultanée par la maladie de Gumboro et la coccidiose chez le poulet de race locale. Etude d'un cas. Revue Méd. trop., 42 (3) : 330.

6. UGOCHUKWU E.I., 1982 : Coccidiose cæcale chez le poulet après une vaccination intramusculaire contre la maladie de Newcastle. Bull. Santé Prod. Anim. Afr., 30 (47) : 397-401.

7. WILLIAM R.B., 1995. Epidemiological studies of coccidiosis in the domesticated fowl (Gallus gallus): IV. Reciprocity between the immune status of floor-reared chickens and their excretion of oocysts. Appl. Parasitol., 36 (4): 290-298

Reçu le 19.11.96, accepté le 12.6.97 


\section{Summary}

Maho A., N dobale B. Occurrence of caecal coccidiosis in laying hens following various experiments

Several experiments were conducted the same day using Lohmann brown laying hens from a farm located at the periphery of Ndjamena. A $6.6 \%$ mortality rate was recorded six days later. Post-mortem examinations, parasitological and microbiological analyses helped identify Eimeria tenella as the causal agent. In conclusion, the combined effects of these experiments have decreased the resistance of the birds and induced the onset of the disease.

Key words: Poultry - Layer chicken - Coccidiosis - Eimeria tenella - M ortality - Chad.

\section{Resumen}

Maho A., Ndobale B. Caso de coccidiosis cecal en gallinas ponedoras después de diversas manipulaciones

Seis días después de varias manipulaciones efectuadas el mismo día, sobre un grupo de gallinas ponedoras Lohman café, en una finca situada en los suburbios de N'Djamena, se registró una mortalidad de $6,6 \%$. Las autopsias y los análisis parasitológicos y bacteriológicos permitieron la identificación de Eimeria tenella como responsable y llevaron a la conclusión de que los efectos asociados de esas manipulaciones disminuyeron la resistencia de las aves, provocando la aparición de esta patología.

Palabras clave: Ave de corral - Gallina ponedora Coccidiosis - Eimeria tenella - Mortalidad - Chad. 